\title{
A randomized trial in the investigation of anxiety and depression in patients with coronavirus disease 2019 (COVID-19)
}

\author{
Feng Zhou ${ }^{1,2,3 \#}$, Rong-Rong Wang ${ }^{3,4 \#}$, Han-Ping Huang ${ }^{3}$, Chun-Ling $\mathrm{Du}^{2}$, Chao-Min $\mathrm{Wu}^{2,3}$, \\ Xue-Mei Qian ${ }^{3,5}$, Wei-Li Li ${ }^{3,6}$, Ju-Li Wang ${ }^{2,3}$, Lv-Yan Jiang, ${ }^{3,7}$ Hui-Jia Jiang, ${ }^{3,8}$, Wen-Jie Yu ${ }^{3,9}$, \\ Ke-Bin Cheng ${ }^{10}$
}

${ }^{1}$ Department of Respiratory Medicine, The First Affiliated Hospital of Soochow University, Suzhou, China; ${ }^{2}$ Department of Respiratory Disease, Qingpu Branch of Zhongshan Hospital Affiliated to Fudan University, Shanghai, China; ${ }^{3}$ Department of Infectious Disease, Jinyintan Hospital of Wuhan, Wuhan, China; ${ }^{4}$ Department of Surgical Intensive Care Unit, Qingpu Branch of Zhongshan Hospital Affiliated to Fudan University, Shanghai, China; ${ }^{5}$ Department of Infectious Disease, Qingpu Branch of Zhongshan Hospital Affiliated to Fudan University, Shanghai, China; ${ }^{6}$ Department of Internal Medicine, People's Hospital of Zhujiajiao, Shanghai, China; ${ }^{7}$ Department of Pediatrics, Fengxian Central Hospital, Shanghai, China; ${ }^{8}$ Department of Orthopedics, Fengxian Central Hospital, Shanghai, China; ${ }^{9}$ Department of Orthopedics, Sijing Hospital of Songjiang, Shanghai, China; ${ }^{10}$ Department of Respiratory and Critical Care Medicine, Shanghai Pulmonary Hospital, Tongji University School of Medicine, Shanghai, China

Contributions: (I) Conception and design: F Zhou, KB Cheng; (II) Administrative support: HP Huang, CL Du; (III) Provision of study materials or patients: CM Wu, XM Qian, WL Li; (IV) Collection and assembly of data: RR Wang, JL Wang, LY Jiang, HJ Jiang, WJ Yu; (V) Data analysis and interpretation: F Zhou, RR Wang, KB Cheng; (VI) Manuscript writing: All authors; (VII) Final approval of manuscript: All authors.

\#These authors contributed equally to this work.

Correspondence to: Ke-Bin Cheng. Department of Respiratory and Critical Care Medicine, Shanghai Pulmonary Hospital, Tongji University School of Medicine, 507 Zhengmin Road, Shanghai 200433, China. Email: chengkebin2017@163.com.

Background In March 2020, the World Health Organization (WHO) declared COVID-19 a public health emergency of international concern. A small proportion of patients infected with COVID-19 go on to develop pneumonia. We speculated that COVID-19 may be likely to result in psychological disorders such as anxiety and depression. In this study, we conducted an investigation of anxiety and depression in patients with COVID-19.

Methods: Sixty-five COVID-19 patients were randomly enrolled into this study. Anxiety and depression among participants were measured through the completion of anonymous Chinese-language Zung selfrating anxiety scale and self-rating depression scale questionnaires. Data were analyzed using independent samples $t$-tests, Mann-Whitney U-tests, and $\chi^{2}$ tests.

Results: The questionnaire results showed that $26.15 \%$ and $41.54 \%$ of participants suffered from anxiety and depression, respectively, although there was no significantly statistical difference between the proportions of COVID-19 patients with anxiety and depression. Statistically significant differences in employment status, partial pressure of oxygen, and corticosteroid application existed between moderate- and severe COVID-19 patients $(\mathrm{P}<0.05)$. In particular, the partial pressure of oxygen was significantly lower in severe COVID-19 patients than in their moderate counter parts $(71.31 \pm 23.54$ ss. $101.06 \pm 34.43, \mathrm{U}=156, \mathrm{P}=0.006)$. Total lymphocytes was lower in severe group than in moderate group $[1.659 \pm 0.643$ vs. $0.745(0.645,0.928), \mathrm{U}=109$, $\mathrm{P}=0.000]$. Also, a higher proportion of female than male patients had anxiety $\left(\chi^{2}=5.388, \mathrm{P}=0.02\right)$. COVID-19 patients who received antiviral medications also displayed a higher rate of anxiety $\left(\chi^{2}=4.481, \mathrm{P}=0.034\right)$. Total lymphocytes between the non-anxiety and anxiety had statistical difference $(\mathrm{U}=321, \mathrm{P}=0.019)$. Meanwhile, total lymphocytes between the non-depression and depression also had statistical difference ( $\mathrm{U}=389.5$, $\mathrm{P}=0.01)$.

\footnotetext{
$\wedge$ ORCID: 0000-0002-3261-3915.
} 
Conclusions: Among patients with COVID-19, females and those treated with antiviral medications were more likely to experience anxiety. In addition, our findings reflected the effect of anxiety and depression on immune system.

Keywords! Coronavirus disease 2019 (COVID-19); anxiety; depression; arterial partial pressure of oxygen (arterial $\left.\mathrm{PaO}_{2}\right)$; corticosteroids; antiviral medication

Submitted Dec 15, 2020. Accepted for publication Feb 04, 2021.

doi: 10.21037/apm-21-212

View this article at: http://dx.doi.org/10.21037/apm-21-212

\section{Introduction}

The coronavirus disease 2019 (COVID-19) has been declared a public health emergency of international concern by the World Health Organization (1,2). Globally, as of 9 January 2021, there have been $87,589,206$ confirmed cases of COVID-19 (2).

The clinical manifestations of COVID-19 include fever, nonproductive cough, dyspnea, myalgia, fatigue, normal or decreased leukocyte count, and radiographic evidence of pneumonia. In severe cases, organ dysfunction [e.g., shock, acute respiratory distress syndrome (ARDS), acute cardiac injury, and acute kidney injury] and even death can occur (3). Studies have shown that community-acquired pneumonia (CAP) is often accompanied by anxiety and depression (4). Given the rapid spread of COVID-19 and its severe clinical consequences including severe pneumonia and ARDS, we speculated that COVID-19 may be likely to result in psychological disorders such as anxiety and depression. In addition, with the outbreak of the COVID-19 pandemic, our daily routines were altered. Information about the COVID-19 pandemic overloaded. Potential financial pressure and the risk of social isolation were also the adverse consequences of the COVID-19 pandemic. These factors are main causes leading to anxiety and depression in patients with COVID-19 (5). The manifestations of anxiety and depression in patients with COVID-19 include physical and behavioral changes and emotional disorders, respectively. Physical and behavioral changes manifest feeling fatigued easily, difficulty concentrating or recalling, decreased energy, chronic fatigue, or feeling sluggish frequently, and so on. Emotional symptoms of anxiety and depression include: restlessness, irritability, or feeling on edge, difficulty controlling worry or fear, loss of interest or no longer finding pleasure in activities or hobbies, persistent feelings of sadness, etc. (6). However, data on COVID19 's relationship with anxiety and depression are limited.
Especially, Antiviral treatment is indispensable because COVID-19 is a kind of viral infection disease. The mental side effects of antiviral medications are unclear so far. Data on anxiety and depression caused by antiviral treatment are also limited. In this study, we investigated anxiety and depression in patients with COVID-19 admitted to Jinyintan Hospital, (Wuhan, Hubei).

We present the following article in according with the CONSORT reporting checklist (available at http://dx.doi. org/10.21037/apm-21-212).

\section{Methods}

\section{Participants}

Sixty-five COVID-19 patients who were admitted to the Northern $2^{\text {nd }}, 3^{\text {rd }}$, and $4^{\text {th }}$ wards of Jinyintan Hospital (Wuhan, Hubei) between February and March 2020 were randomly enrolled into this study. All patients were confirmed with COVID-19 in line with the criteria of the Diagnosis and Treatment Protocol for Novel Coronavirus Pneumonia (Trial Version 7) issued by National Health Commission of the People's Republic of China (7). Among the 65 patients were 31 men and 34 women with a mean age of 57.42 years (range, 21-79 years). There were no significant differences in sex or age among these men and women patients. The patients completed anonymous Chinese-version Zung self-rating anxiety scale (SAS) (8) and self-rating depression scale (SDS) (9) questionnaires. Approval for the study was granted by the Ethics Committee of Jinyintan Hospital (No. KY-202045.01). The study was conducted in accordance with the Declaration of Helsinki (as revised in 2013). Verbal consent was obtained from all participations in this study.

The study inclusion criteria were as follow: (I) having a definite diagnosis of COVID-19 based on the Diagnosis and Treatment Protocol for Novel Coronavirus Pneumonia 
(Trial Version 7), (II) being aged between 18 and 80 years, (III) having adequate intellectual ability and educational background, (IV) having been informed and given verbal consent for participation in this study.

The following patients were excluded from the study: (I) pregnant or lactating women; (II) patients with cognitive impairment; (III) patients with aphasia or memory disorders; (IV) patients with mental illness; (V) patients taking anti-anxiety or antidepressants for a long time; (VI) patients with alcohol or drug abuse.

\section{Measures used}

Anxiety and depression were measured using the SAS (Selfrating Anxiety Scale) and SDS (Self-rating Depression Scale), respectively. Compiled by Zung in 1971, the SAS is a 20-item, self-reporting measure of anxiety symptoms. Each of the items is ranked on a 4-point Likert scale, ranging from "never occurring" and "some of the time" to "most of the time". Responses are summed to calculate a total score, with a higher score indicating a greater level of anxious symptomatology. High SAS scores indicate severe anxiety $(\mathrm{SAS}>69)$, while low scores (SAS <50) are a sign of a normal emotional state (10). The SDS is a 20 -item self-reporting tool, which was also developed by Zung, to measure depressive symptoms and for depression screening. It consists of 20 items, covering emotional, psychological, and physical symptoms. The patient describes how often they experience a particular symptom ("not at all", "sometimes", "often", or "always"). The answers correspond to a specific score (not at all: 1; sometimes: 2; often: 3; and always: 4), and the total score helps in the assessment of depression symptoms. The severity of depression according to the SDS was then calculated and used for statistical analysis. High SDS scores indicate severe depression (SDS >72), whereas low scores (SDS <53) are a sign of a normal emotional state (11). The SAS and SDS are both reliable and valid and are suitable for clinical and research use with satisfactory properties $(10,11)$.

\section{Statistical analysis}

Continuous variables were expressed as mean \pm standard deviation (SD), or median [interquartile range (IQR)], and were compared with the independent samples $t$-test or the Mann-Whitney $U$-test (as appropriate). The comparison of categorical variables, which were expressed as frequencies (percentages), was performed using $\chi^{2}$ test. Statistical analyses were conducted using the statistical package SPSS 16.0 (SPSS Inc, Chicago, IL, USA). $\mathrm{P}<0.05$ was considered to be statistically significant.

\section{Results}

\section{Participant characteristics}

A total of 65 COVID-19 patients were enrolled and divided into 2 groups according to the disease severity (7): the moderate group and the severe group (typically defined by clinical manifestations including respiratory distress or hypoxia). In our study, we got the data of arterial partial pressure of oxygen $\left(\mathrm{PaO}_{2}\right)$, which was helpful to calculate the data of saturation oxygen $\left(\mathrm{SaO}_{2}\right)$ and arterial partial pressure of oxygen $\left(\mathrm{PaO}_{2}\right)$ /fraction of inspired oxygen $\left(\mathrm{FiO}_{2}\right)$. These data could identify the moderate and severe COVID-19. The moderate and severe groups consisted of 53 patients and 12 patients, respectively. The demographic, clinical, and laboratory characteristics of moderate and severe COVID-19 patients are shown in Table 1. Statistically significant differences existed in employment status, partial pressure of oxygen $\left(\mathrm{PaO}_{2}\right)$, and corticosteroid application between the moderate and severe COVID-19 groups $(\mathrm{P}<0.05)$. In particular, the $\mathrm{PaO}_{2}$ of the severe COVID-19 patients was lower than that of the moderate COVID-19 patients $(71.31 \pm 23.54$ vs. $101.06 \pm 34.43, U=156, \mathrm{P}=0.006)$. Additionally, total lymphocytes were lower in severe group than in moderate group (1.659 0.643 vs. 0.745 (0.645,0.928), $U=109$, $\mathrm{P}=0.000)$. No significant differences were found between the 2 groups in terms of age, sex, duration of education, family cluster situation, use of antiviral medication, history of chronic diseases, IL-6, SAS score, or SDS score $(\mathrm{P}>0.05)$.

\section{Differences between COVID-19 patients with and without anxiety}

According to SAS score, the 65 COVID-19 patients were divided into 2 groups: the non-anxiety group and the anxiety group. Forty-eight COVID-19 patients were in the non-anxiety group and 17 COVID-19 patients were in the anxiety group. As shown in Table 2, demographic, clinical, and laboratory variables were compared between COVID-19 patients with and without anxiety. There was significantly statistical difference in sex between the anxiety and non-anxiety groups $(\mathrm{P}<0.05)$. In addition, distinct statistical difference in the use of antiviral medication 
Table 1 Demographic and clinical characteristics of 65 COVID-19 patients

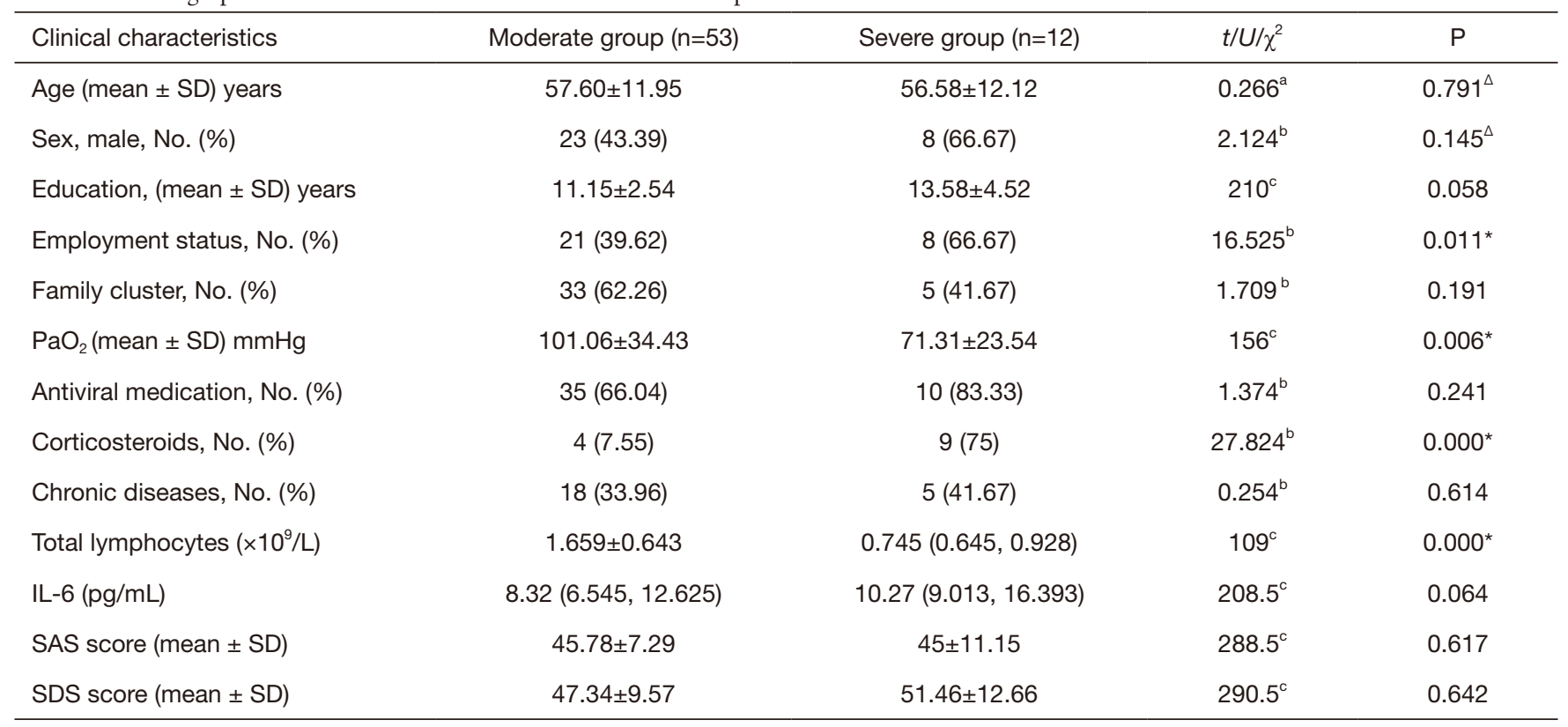

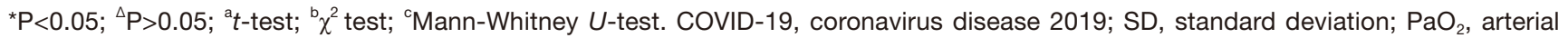
partial pressure of oxygen; SAS, self-rating anxiety scale; SDS, self-rating depression scale.

Table 2 Comparison of the clinical characteristics of COVID-19 patients with and without anxiety

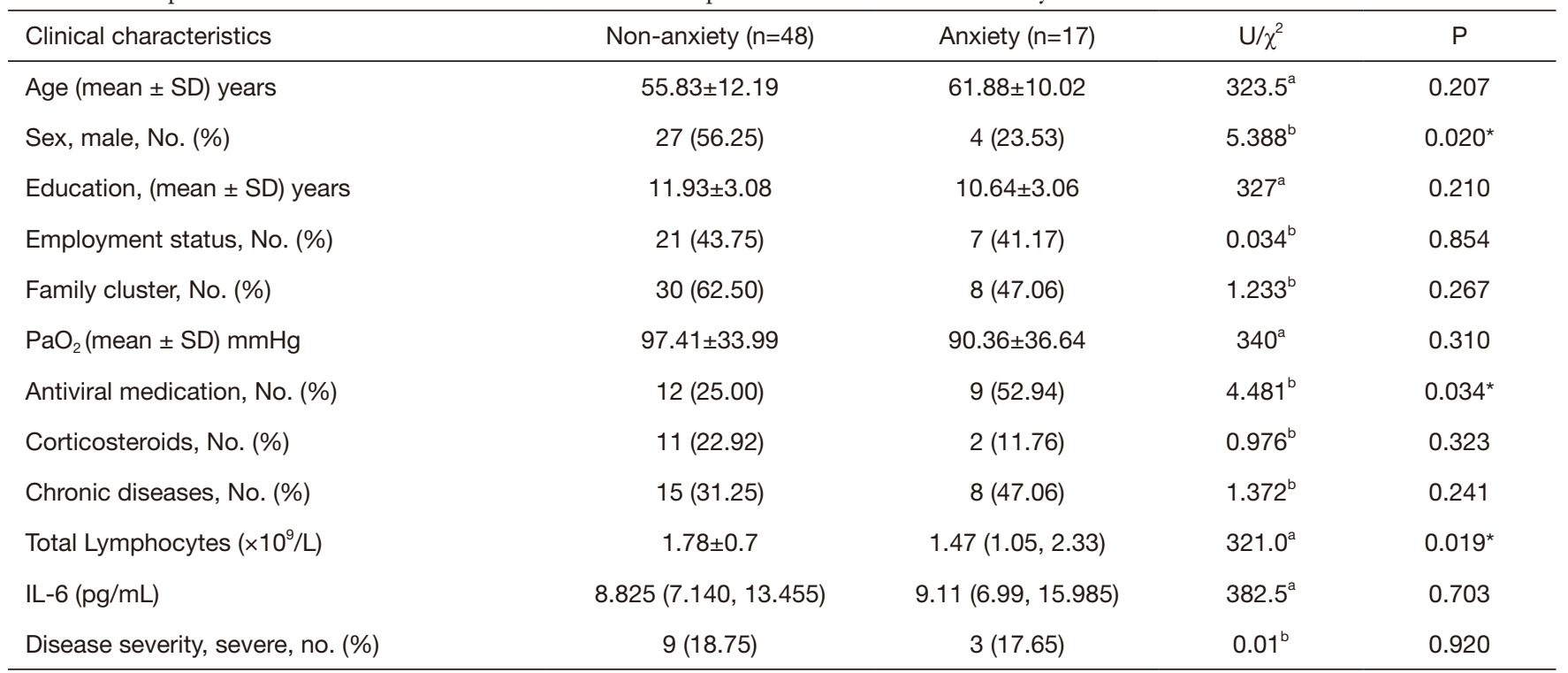

${ }^{*} \mathrm{P}<0.05$; ${ }^{a}$ Mann-Whitney $U$-test; ${ }^{b} \chi^{2}$ test. COVID-19, coronavirus disease 2019; SD, standard deviation; $\mathrm{PaO}_{2}$, arterial partial pressure of oxygen.

was found between the anxiety and non-anxiety groups $(\mathrm{P}<0.05)$. Meanwhile, there was statistical difference of total lymphocytes between the non-anxiety and anxiety groups [1.78 \pm 0.7 vs. 1.47 (1.05, 2.33), $U=321, \mathrm{P}=0.019]$. However, no statistically significant differences were found in age, duration of education, employment status, family cluster situation, $\mathrm{PaO}_{2}$, use of corticosteroid treatment, history of chronic diseases, IL-6 or disease severity between the 2 
Table 3 Comparison of the clinical characteristics of COVID-19 patients with and without depression

\begin{tabular}{|c|c|c|c|c|}
\hline Clinical characteristics & Non-depression $(\mathrm{n}=38)$ & Depression $(n=27)$ & $\mathrm{U} / \chi^{2}$ & $\mathrm{P}$ \\
\hline Age $($ mean $\pm S D)$ years & $56.45 \pm 12.85$ & $58.78 \pm 10.48$ & $495.5^{\mathrm{a}}$ & 0.816 \\
\hline Sex, male, No. (\%) & $21(55.26)$ & $10(37.04)$ & $2.102^{b}$ & 0.147 \\
\hline Education, (mean $\pm \mathrm{SD}$ ) years & $12.32 \pm 2.96$ & $10.59 \pm 3.08$ & $373^{a}$ & 0.053 \\
\hline Employment status, No. (\%) & $15(39.47)$ & $13(48.15)$ & $0.484^{b}$ & 0.486 \\
\hline $\mathrm{PaO}_{2}($ mean $\pm \mathrm{SD}) \mathrm{mmHg}$ & $96.24 \pm 37.69$ & $94.62 \pm 30.26$ & $492^{a}$ & 0.780 \\
\hline Antiviral medication, No. (\%) & $28(73.68)$ & $18(66.67)$ & $0.376^{b}$ & 0.540 \\
\hline Corticosteroids, No. (\%) & $8(21.05)$ & $5(18.52)$ & $0.063^{b}$ & 0.801 \\
\hline Chronic Diseases, No. (\%) & $12(31.58)$ & $11(40.74)$ & $0.579^{b}$ & 0.447 \\
\hline
\end{tabular}

${ }^{*} \mathrm{P}<0.05$; ${ }^{a}$ Mann-Whitney $U$-test; ${ }^{\mathrm{b}} \chi^{2}$ test. COVID-19, coronavirus disease 2019; SD, standard deviation; $\mathrm{PaO}_{2}$, arterial partial pressure of oxygen.

Table 4 Comparison in the proportions of COVID-19 patients with anxiety and depression

\begin{tabular}{lcccc}
\hline & Anxiety $(n=17)$ & Depression $(n=27)$ & $\chi^{2}$ & $P$ \\
\hline Percentage & $26.15 \%$ & $41.54 \%$ & 3.436 & 0.064 \\
\hline
\end{tabular}

COVID-19, coronavirus disease 2019.

groups $(\mathrm{P}>0.05)$.

\section{Differences between COVID-19 patients with and without depression}

The 65 COVID-19 patients were divided into 2 groups (the depression and non-depression groups) according to their SDS scores. There were 38 and 27 COVID-19 patients in the non-depression and depression groups, respectively. As can be seen in Table 3, no statistical differences were found in most of the clinical characteristic indexes between the 2 groups $(\mathrm{P}>0.05)$. However, there was significant statistical difference of total lymphocytes between the non-depression and depression groups $[1.79 \pm 0.73$ vs. 1.43 (1.14, 2.25), $U=389.5, \mathrm{P}=0.01]$.

\section{Difference in the proportions of COVID-19 patients with anxiety and depression}

As shown in Table 4, there were 17 (26.15\%) patients with anxiety and 27 (41.54\%) patients with depression among the 65 COVID-19 patients enrolled into the study. However, there was no statistical difference between the proportions of patients with anxiety and depression in the cohort $\left(\chi^{2}=3.436, \mathrm{P}=0.064\right)$.

\section{Discussion}

COVID-19 has now spread to every continent except Antarctica. As the global pandemic rumbles on, we are constantly being updated on the virus and the latest death tolls. Understandably, this can be overwhelming. COVID-19 is highly contagious and can quickly progress to pneumonia, and in severe cases, ARDS and even death (12). With COVID-19's rapid spread since December 2019, as well as its potentially grave clinical outcomes, it is entirely reasonable to believe that this disease has impacted the mental health of individuals, especially those who have contracted it, resulting in psychological disorders such as anxiety and depression (13). 
It is notable that during the outbreak of severe acute respiratory syndrome in 2003, some patients with the disease were found to have mental disorders (14). In our study of 65 patients with COVID-19, we found 17 patients (26.15\%) to have anxiety and 27 patients $(41.54 \%)$ to have depression. This result illustrates that severe contagious diseases can cause psychological disorders in the patients who suffer them.

In our study, patients with moderate and severe COVID-19, classified according to Chinese diagnostic and treatment criteria, were compared (7). There were no significant differences in age, sex, duration of education, family cluster situation, use of antiviral medication, history of chronic diseases, SAS score, or SDS score between the 2 groups $(\mathrm{P}>0.05)$. However, employment status did show a significant statistical difference: employed patients displayed more serious clinical manifestations and were more likely to have severe disease than unemployed patients $\left(\chi^{2}=16.525, \mathrm{P}=0.011\right)$. This observation may be attributable to employed individuals having a higher probability of close contact with other people than those who are unemployed. Moreover, due to the pressure and high speed of working life in modern society, the health of many employees is subpar. In Price's study (15), the employed population was reported to have a higher prevalence of heart disease and a more severe disease situation. By analogy, the employed population had a higher risk of disease. As stated in this study, the employed population was at a higher risk of contracting COVID-19. Furthermore, we also found that the severe COVID-19 group had a lower $\mathrm{PaO}_{2}$ level and a higher rate of corticosteroid use. These results were consistent with those of recently issued clinical research on COVID-19 (16,17). We focused on arterial partial pressure of oxygen $\left(\mathrm{PaO}_{2}\right)$ due to it was an important index in the classification of the severity of COVID-19. In our study, the results about severe group of COVID-19 having lower $\mathrm{PaO}_{2}$ level were consistent with the criteria of clinical classification according to Diagnosis and Treatment Protocol for Novel Coronavirus Pneumonia (7). Moreover, corticostiroids played a key role in treating severe patients with COVID-19, especially in reducing the death of risk (18). There were higher rate of corticostiroids in severe group than in moderate group with COVID-19 in our study. This result was in keep with the above mentioned research (18). Apart from the above reasons, we focused on $\mathrm{PaO}_{2}$ and corticostiroids in patients with COVID-19 because of the relationtionship between mental disorders, including anxiety and depression, and hypoxia and higher dose of corticostiroids. Some researches $(19,20)$ stated that hypoxia, also called as lower level of $\mathrm{PaO}_{2}$, and higher dose of corticostiroids would lead to anxiety and depression. In our study, there weren't any significant statistical difference between $\mathrm{PaO}_{2}$, corticostiroids and anxiety, depression. It meaned that further researches needed to be done in the future.

Seeing from Table 1, total lymphocytes in severe group of patients with COVID-19 was lower than in moderate group. Meanwhile, total lymphocytes were statistically difference between non-anxiety and anxiety or between non-depression and depression in patients with COVID-19 as shown in Tables 2 and 3. These findings of our study and other researches $(21,22)$ reflected the effect of disease severity, anxiety and depression on immunity system.

Our analysis of anxiety among the patients revealed that a higher proportion of females were affected than males $\left(\chi^{2}=5.388, \mathrm{P}=0.02\right)$. Anxiety maybe a more common problem for women than it is for men. While this may, in part, be due to cultural and social traditions and expectations, it is also related to the chemicals present in the female body, as well as the myriad of physical changes that occur in women's bodies compared to men's. Furthermore, some studies have reported that women are more likely to experience emotional disorders than men $(23,24)$. Additionally, in our study, those COVID-19 patients who were treated with antiviral medications had higher rate of anxiety $\left(\chi^{2}=4.481, \mathrm{P}=0.034\right)$. Antiviral medications used to treat patients in our study included ribavirin, Kaletra (lopinavir and ritonavir), Arbidol, and oseltamivir. Some studies have also found that the above mentioned antiviral medications can have mental side effects such as anxiety (25-28), which suggests that we must pay close attention to changes in the mental state of COVID-19 patients when administering these medications.

No significant statistical differences were found between COVID-19 patients with and without depression, or in the proportions of COVID-19 patients with anxiety and depression, which is due to the small number of patients in the study. Thus, as stated above, more relevant studies need to be conducted to support our hypothesis.

Though some patients with COVID-19 experienced anxiety and depression, there were several approaches to alleviate these adverse effects including taking deep breaths, stretch or mediate, trying to eat healthy, well balanced meals, to exercise regularly, to avoid excessive alcohol and tobacco, to connect with loved ones through phone calls or 
video chats (29).

\section{Conclusions}

In this study, we investigated anxiety and depression among 65 COVID-19 patients admitted to the Northern $2^{\text {nd }}, 3^{\text {rd }}$, and $4^{\text {th }}$ wards of Jinyintan Hospital in Wuhan, Hubei. We found that employed patients were more likely to have severe COVID-19 than unemployed patients. In addition, the index of $\mathrm{PaO}_{2}$ and the total lymphocytes in patients with severe COVID-19 patients was lower than that in moderate patients, whereas the dose of corticosteroids was higher. These characteristics were consistent with the clinical and laboratory manifestations and treatment regimens of COVID-19. We also found that female patients with COVID-19 were more likely to experience anxiety, as were those patients treated with antiviral medications. Besides of these findings, we also discovered the effect of anxiety and depression on immunity system from the statistical difference of total lymphocytes between the group of non-anxiety and anxiety and between the group of nondepression and depression.

\section{Acknowledgments}

Funding: This work was supported by grants from the Science and Technology Commission of Qingpu District, Shanghai (QKY2018-12).

\section{Footnote}

Reporting Checklist: The authors have completed the CONSORT reporting checklist. Available at http://dx.doi. org/10.21037/apm-21-212

Data Sharing Statement: Available at http://dx.doi. org/10.21037/apm-21-212

Conflicts of Interest: All authors have completed the ICMJE uniform disclosure form (available at http://dx.doi. org/10.21037/apm-21-212). The authors have no conflicts of interest to declare.

Ethical Statement: The authors are accountable for all aspects of the work in ensuring that questions related to the accuracy or integrity of any part of the work are appropriately investigated and resolved. Approval for the study was granted by the Ethics Committee of Jinyintan
Hospital (No. KY-2020-45.01). The study was conducted in accordance with the Declaration of Helsinki (as revised in 2013). Verbal consent was obtained from all participations in this study.

Open Access Statement: This is an Open Access article distributed in accordance with the Creative Commons Attribution-NonCommercial-NoDerivs 4.0 International License (CC BY-NC-ND 4.0), which permits the noncommercial replication and distribution of the article with the strict proviso that no changes or edits are made and the original work is properly cited (including links to both the formal publication through the relevant DOI and the license). See: https://creativecommons.org/licenses/by-nc-nd/4.0/.

\section{References}

1. Paules CI, Marston HD, Fauci AS. Coronavirus Infections-More Than Just the Common Cold. JAMA 2020;323:707-8.

2. World Health Organization. WHO Coronavirus Disease (COVID-19) Dashboard 2021. Available online: https:// covid19.who.int/

3. Huang C, Wang Y, Li X, et al. Clinical features of patients infected with 2019 novel coronavirus in Wuhan, China. Lancet 2020;395:497-506.

4. Kao LT, Liu SP, Lin HC, et al. Poor clinical outcomes among pneumonia patients with depressive disorder. PLoS One 2014;9:e116436.

5. MAYO CLINIC. COVID-19 and your mental health 2020. Available online: https://www.mayoclinic.org/ diseases-conditions/coronavirus/in-depth/mental-healthcovid-19/art-20482731

6. HEALTHLINE. Depression and Anxiety: How to Identify and Treat Coexisting Symptoms 2018 Available online: https://www.healthline.com/health/mental-health/ depression-and-anxiety

7. National Health Commission \& State Administration of Traditional Chinese Medicine. Diagnosis and Treatment Protocol for Novel Coronavirus Pneumonia (Trial Version 7). Chinese Medical Journal 2020;133:1087-95.

8. Zung WW. A rating instrument for anxiety disorders. Psychosomatics 1971;12:371-9.

9. Zung WW. A Self-Rating Depression Scale. Arch Gen Psychiatry 1965;12:63-70.

10. Ramirez SZ, Lukenbill J. Psychometric Properties of the Zung Self-Rating Anxiety Scale for Adults with Intellectual Disabilities (SAS-ID). J Dev Phys Disabil 2008;20:573-80. 
11. Fountoulakis KN, lacovides A, Samolis S, et al. Reliability, validity and psychometric properties of the Greek translation of the Zung Depression Rating Scale. BMC Psychiatry 2001;1:6.

12. Wu C, Chen X, Cai Y, et al. Risk Factors Associated With Acute Respiratory Distress Syndrome and Death in Patients With Coronavirus Disease 2019 Pneumonia in Wuhan, China. JAMA Intern Med 2020;180:934-43.

13. Pfefferbaum B, North CS. Mental Health and the Covid-19 Pandemic. N Engl J Med 2020;383:510-2.

14. Wu KK, Chan SK, Ma TM. Posttraumatic stress, anxiety, and depression in survivors of severe acute respiratory syndrome (SARS). J Trauma Stress 2005;18:39-42.

15. Price AE. Heart disease and work. Heart 2004;90:1077-84.

16. Liu K, Fang YY, Deng Y, et al. Clinical characteristics of novel coronavirus cases in tertiary hospitals in Hubei Province. Chin Med J (Engl) 2020;133:1025-31.

17. Guan WJ, Ni ZY, Hu Y, et al. Clinical Characteristics of Coronavirus Disease 2019 in China. N Engl J Med 2020;382:1708-20.

18. WHO Rapid Evidence Appraisal for COVID-19 Therapies (REACT) Working Group, Sterne JAC, Murthy S, et al. Association Between Administration of Systemic Corticosteroids and Mortality Among Critically Ill Patients With COVID-19: A Meta-analysis. JAMA 2020;324:1330-41.

19. Zhao F, Yang J, Cui R. Effect of Hypoxic Injury in Mood Disorder. Neural Plasticity 2017;2017:6986983.

20. Pretorius E. Corticosteroids, depression and the role of serotonin. Rev Neurosci 2004;15:109-16.

Cite this article as: Zhou F, Wang RR, Huang HP, Du CL, Wu CM, Qian XM, Li WL, Wang JL, Jiang LY, Jiang HJ, Yu WJ, Cheng KB. Investigation of anxiety and depression in patients with coronavirus disease 2019 (COVID-19). Ann Palliat Med 2021;10(2):2167-2174. doi: 10.21037/apm-21-212
21. Wagner J, DuPont A, Larson S, et al. Absolute lymphocyte count is a prognostic marker in Covid-19: A retrospective cohort review. Int J Lab Hematol 2020;42:761-5.

22. Dalar L, Sibel Ü, Karasulu AL, et al. The Relationship Between Lymphocytes and Anxiety and Depression in Pulmonary Tuberculosis. Turk Thorac J 2010;11:112-6.

23. Donner NC, Lowry CA. Sex differences in anxiety and emotional behavior. Pflugers Arch 2013;465:601-26.

24. McLean CP, Asnaani A, Litz BT, et al. Gender differences in anxiety disorders: prevalence, course of illness, comorbidity and burden of illness. J Psychiatr Res 2011;45:1027-35.

25. Koren G, King S, Knowles S, et al. Ribavirin in the treatment of SARS: A new trick for an old drug? Cmaj 2003;168:1289-92.

26. Chan KS, Lai ST, Chu CM, et al. Treatment of severe acute respiratory syndrome with lopinavir/ritonavir: a multicentre retrospective matched cohort study. Hong Kong Med J 2003;9:399-406.

27. Boriskin YS, Leneva IA, Pécheur EI, et al. Arbidol: a broad-spectrum antiviral compound that blocks viral fusion. Curr Med Chem 2008;15:997-1005.

28. Razonable RR. Antiviral drugs for viruses other than human immunodeficiency virus. Mayo Clin Proc 2011;86:1009-26.

29. CDC. Coping with Stress 2021. Available online: https:// www.cdc.gov/coronavirus/2019-ncov/daily-life-coping/ managing-stress-anxiety.html

(English Language Editor: J. Reynolds) 